\title{
Effect of Laser Irradiation on the Structural and Electrical Properties of Co-Zn Ferrite Thin Film
}

\author{
Nehad M. Tashtoush \\ Physics Department, Faculty of Science, Mu'tah University, Jordan \\ A sabbatical leave, Physics Department, King Khalid University, Abha, Saudi Arabia
}

\begin{abstract}
Thin films of irradiated and non-irradiated Co-Zn ferrite was prepared by the thermal evaporation method on a glass substrate. The samples were annealed at $200^{\circ} \mathrm{C}, 400^{\circ} \mathrm{C}$ and $600^{\circ} \mathrm{C}$ for $1 \mathrm{hr}$. The XRD analysis of all kinds of films, prepared for this study, shows that the spinel phase is almost completed after annealing. The change of the XRD after annealing is explained by the change of the relative quantities of the various magnetic phases (spinel and oxides) present before and after the annealing process. After annealing at $600^{\circ} \mathrm{C}$, the material shows a spinel phase with lattice constant $8.30 \mathrm{~A}^{\circ}$ for normal samples and $8.38 \mathrm{~A}^{\circ}$ for the irradiated ones, respectively. After laser irradiation, the XRD pattern indicates the formation of a distorted spinel structure besides the spinel phase. A metallic behavior with temperature was observed in the two samples, irradiated and non-irradiated. The resistance of the sample was found to decrease by annealing process which may be due to the decrease in the number of grain boundaries.
\end{abstract}

Key words: Electrical properties, CoZn ferrite, laser irradiation, CoZn thin film

\section{INTRODUCTION}

Until recently the most experiments made on ferrites have been performed on bulk single crystal or polycrystalline material due to the difficulties of producing high-quality thin film samples. Flux melts, liquid phase epitaxy $\mathrm{LPE}^{[1]}$ and the sputtering techniques have been used in the past to prepare thin film of ferrites ${ }^{[2]}$.

It was shown that the film of spinel ferrite can be formed by using the pulsed laser deposition technique $^{[3,4,5]}$. The experimental result, illustrates the change of XRD patterns upon annealing at high temperatures. Such changes are explained by the change of the relative quantities of the various magnetic phases (spinel and oxides) present in the sample due to a different annealing. Recently, a number of materials have been investigated for applications as thin film high density recording media ${ }^{[6]}$. In addition to their chemical stability and mechanical hardness, the thin film ferrite media have been shown to exhibit low recording noise ${ }^{[7-9]}$. In thin ferrite films it is important to control the crystallographic texture, microstructure, and magnetic properties for such applications. The properties of these thin films can be significantly varied by changing the deposition conditions. The growth of thin layers of magnetically soft ferrites such as $\mathrm{Mn}-\mathrm{Zn}$ or Ni-Zn ferrite has been studied recently by various groups ${ }^{[10-14]}$. The effect of low intensity laser beam on the $\mathrm{Mn}-\mathrm{Zn}$ Ferrite thin film was studied ${ }^{[12]}$. These ferrite thin films were grown by the method of chemical vapor deposition and were deposited onto (110) $\mathrm{MnO}$ crystals. Soft magnetic MnZn ferrite thin films with high electrical resistivity were studied in developing micro-inductors and micro-transformers ${ }^{[13]}$.

There are no any attempts to study the effect of laser irradiation on the structural and electrical resistance of Co-Zn thin film ferrite. Therefore, in the present study, we attempt to explore the effect of laser irradiation on the electrical and structural properties of Co-Zn ferrite thin films, which are very important for technological applications.

\section{MATERIALS AND METHODS}

The $\mathrm{Co}_{0.6} \mathrm{Zn}_{0.4} \mathrm{Fe}_{2} \mathrm{O}_{4}$ ferrite target was prepared by the double sintering ceramic technique from raw materials of purity $99,999 \%$. The crystalline structure was spinel phase, which was verified using X-ray powder diffraction. The bulk material was irradiated using Nd-Y laser. The average power is 4 watts and the exposure time was 15 minutes. The CoZn ferrite thin film was prepared using a Leybold film evaporator system (Edward coating system E306A). All samples were prepared in the same conditions and were of the same thickness on a glass substrate. The prepared thin films were annealed at $200^{\circ} \mathrm{C}, 400^{\circ} \mathrm{C}$ and $600^{\circ} \mathrm{C}$ for one hour. The XRD for all samples were done by using Philips PW 1729 using $\mathrm{CuK}_{\alpha}$ radiation. The electrodes were made from platinum and silver epoxy was used to connect the electrode with the sample. The electrical resistivity of the films was measured using the fourpoint-probe method in the temperature range from 30 to 180 (C. A current source (Keithley 228A) supplied a 
constant current of $0.01 \mathrm{~A}$ through the sample and the voltage across the probes was measured by Keithley $614 \mathrm{~A}$ electrometer.

\section{RESULTS AND DISCUSSION}

X-ray diffraction pattern for $\mathrm{Co}_{0.6} \mathrm{Zn}_{0.4} \mathrm{Fe}_{2} \mathrm{O}_{4}$ thin film: Figure (1-a) shows the XRD patterns (or spectra) of the as-deposited, irradiated and non-irradiated, thin films of the same thickness. Also, Fig. (1-b, c, d) show the XRD patterns for annealed, irradiated and nonirradiated, thin films at 200,400 and $600^{\circ} \mathrm{C}$ for $1 \mathrm{hr}$. In general, the XRD patterns show that the spinel phase is almost present after annealing temperature $600^{\circ} \mathrm{C}$, for $1 \mathrm{hr}$. Also, For each sample, the XRD after annealing displays very strong difference from XRD before annealing.

By discussing the non-irradiated thin films behavior first, it appears that; as indicated in Fig. (1-a), the as-deposited non-irradiated thin films show two peaks at $2 \theta=43.14$ with high intensity and at $2 \theta=$ 50.36 with low intensity. We think that the two peaks are belonging to the oxide phase $\mathrm{Fe}_{2} \mathrm{O}_{3}$ ( $\alpha$ phase). The largest portion of the two films is still amorphous. However, Fig. (1-b) shows that for the non-irradiated sample and annealed at $200^{\circ} \mathrm{C}$, another important diffraction peak, at $2 \theta=47.23$ from the plane (113) which belongs to $\mathrm{Fe}_{2} \mathrm{O}_{3}$, was appeared (the main characteristic peak of $\mathrm{Fe}_{2} \mathrm{O}_{3}$ is (113). This means that the oxide phase grows with annealing at $200^{\circ} \mathrm{C}$ and is predominant. Moreover, for that non-irradiated and annealed at $400^{\circ} \mathrm{C}$ three diffraction peaks appeared at $2 \theta=14.3,40.8$ and 52.4. The last two peaks belong to the spinel phase structure from the planes (311) and (400) respectively. The lattice parameter of the growing spinel phase of this sample was calculated to be $8.31 \mathrm{~A}$. The non-radiated that annealed at $600^{\circ} \mathrm{C}$ for $1 \mathrm{hr}$ shows many diffraction peaks in the XRD patterns. The peaks that belong to the spinel phase are $311,400,422$ and 511. There are another two peaks 111, 024 appeared and belonging to the oxide phase $\mathrm{Fe}_{3} \mathrm{O}_{4}$ and $\mathrm{Fe}_{2} \mathrm{O}_{3}$ respectively. The percentage of the spinel phase became $70 \%$ of the total phases present. The lattice parameter deduced from this pattern is $8.3 \mathrm{~A}^{\circ}$. This result indicates that the lattice parameter is the same for the sample annealed at 400 and that at $600^{\circ}$ indicting that the annealing process increases the percentage of the spinel phase but not affect the unit cell size. It is also noticed that this lattice parameter becomes closer to that of the bulk material $\mathrm{Co}_{0.6} \mathrm{Zn}_{0.4} \mathrm{Fe}_{2} \mathrm{O}_{4}$, which means that the film become a polycrystalline film after annealing to $600^{\circ} \mathrm{C}$.

For the second kind of thin films (i.e. Irradiated), we notice the existence of three weak peaks in Fig. (1a) for the as-deposited and irradiated thin films. These peaks are at $2 \theta=43.54,52.35$ and at 50.25 from the planes (400), (422) and (024) respectively. These diffraction peaks belong to the oxide phase. The intensity of the peaks is less than that of non-irradiated thin films. However, for irradiated and annealed thin films at $200^{\circ} \mathrm{C}$, Fig. (1-b) show that one distinct peak appears in the XRD patterns at $2 \theta=47.4$ belonging to the oxide phase. Also, Fig. (1-c) shows that the irradiated film and annealed at $400^{\circ} \mathrm{C}$ has three peaks at $2 \theta=19.9,53$ and 64 of the planes 110, 202 and 422 respectively. The first peak is due to the spinel phase whereas the second and third due to the oxide phases. Finally, as indicated in Fig. (1-d), the irradiated film and annealed at $600^{\circ} \mathrm{C}$, the XRD patterns have more diffraction peaks than the non-irradiated one. The appeared peaks were from the planes $111,306,024$, $311,400,422$, and 440 at $2 \theta=18,77,4,8.7,36,42.4$, 52.9 and 63.6 respectively. The first three peaks belong to the oxide phase and the last four peaks are belonging to the spinel phase structure. The intensity of the peaks is different from that for non-irradiated and annealed at 600 (C. There are two other peaks appeared at $2 \theta=22$ and 28 respectively may be due the distorted phase produced by radiation beside the spinel one. The lattice parameter deduced here is $8.38 \mathrm{~A}$ which is higher than that of the non-irradiated one. From the number of peaks appeared in the diffraction and its intensity with a comparison that of the ideal bulk XRD pattern we estimate that the percentage of the spinel phase is $60 \%$ lower than that of non-irradiated sample due to the appearance of the distorted phase.

The XRD spectra of the as deposited Co $\mathrm{Zn}$ ferrite film consists of two phases, the amorphous and the crystalline phase. Upon annealing at $600^{\circ} \mathrm{C}$, the spinel phase is obtained. This is related to the presence of $\mathrm{Zn}$ which is responsible of the amorphous phase formation. A new meta-stable phase is formed upon annealing at $600^{\circ} \mathrm{C}$ for $1 \mathrm{hr}$. The new phase has the spinel structure in which $\mathrm{Fe}$ and $\mathrm{Co}$ ions take orders positions of the octahedral sites whereas $\mathrm{Fe}$ and $\mathrm{Zn}$ ions take ordered positions at tetrahedral sites. In the as deposited Co-Zn ferrite film, the structure of the crystalline is of the close packed planes and shoot kind of order. Upon annealing two things happened; one is that the $\mathrm{Zn}$ atom within each closed packed plane form a tetrahedral ordering pattern with 6 oxygen atoms surrounding each ion. The second is that the packing $\mathrm{ABC}$ direction takes long range packing and forms a spinel phase. In the as deposited film there exist imperfections through the whole thickness of the film. This is in contrast to film after annealing where the formation of small crystallites occurs during the initial stage of growth only. On the left stage of growth a very distinct columnar structure appears and the grain size increases after annealing.

The laser effect is a bulk effect, the structural defects are preserved in the whole sample even after mechanical grinding of the laser-irradiated sample. The structure defect distortion was observed in both the powder polycrystalline and the thin film of the ferrite ${ }^{[23]}$. 


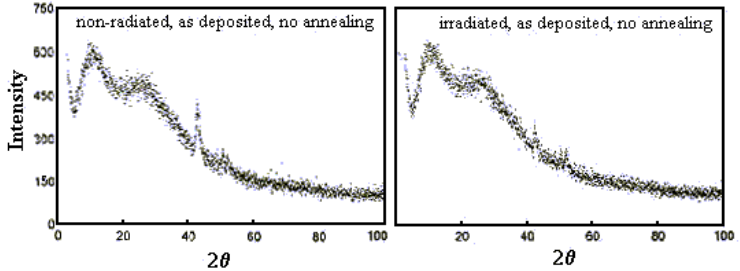

(a)
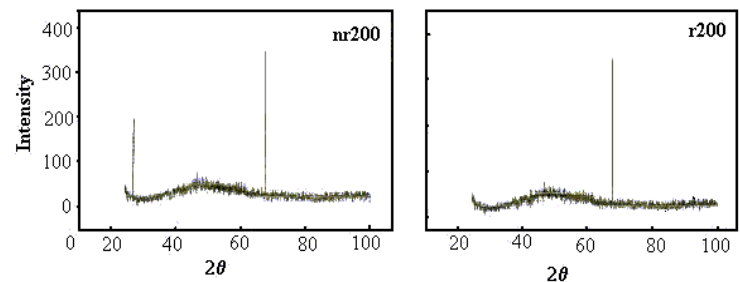

(b)
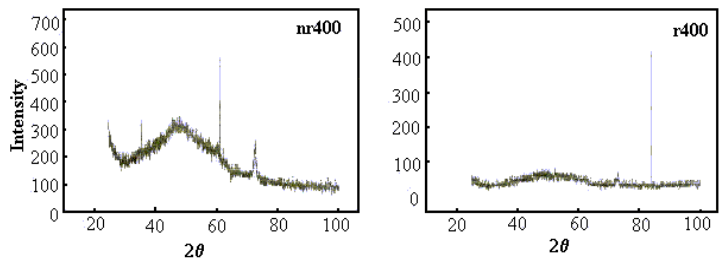

(c)
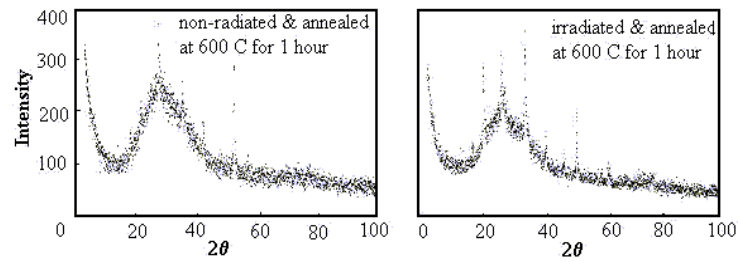

(d)

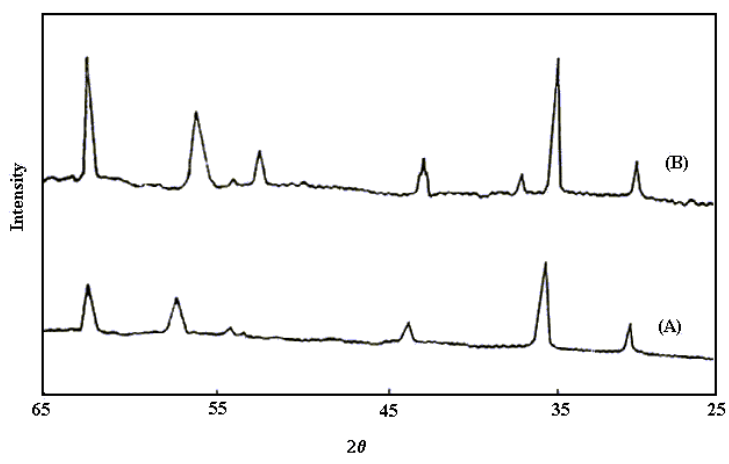

Fig. 2: Bulk ferrite sample (A), thin film sample annealed at $600^{\circ} \mathrm{C}$ for one hour (B).

As seen from the XRD patterns (Fig. 1a-d and Fig. 2) for the films before and after irradiation, the effect of laser irradiation on the films are preserved even after the thermal deposition process of the film.

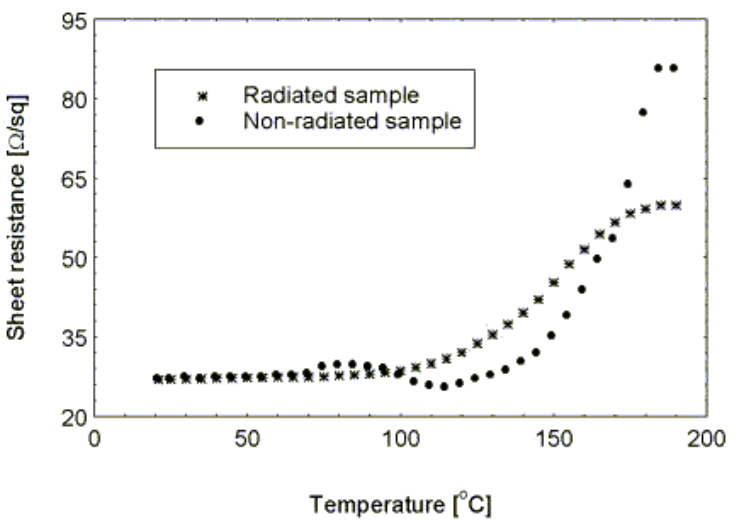

Fig. 3: Sheet resistance of non-radiated (dot) and irradiated (star) thin films as a function of temperature.

The as grown samples show little evidence of the crystalline oxide phase, where a large proportion of the sample appears to be amorphous. After the annealing treatment to $600^{\circ} \mathrm{C}$ for $1 \mathrm{hr}$ the sample displays very clear and strong spinel phase diffraction peaks. This clearly illustrates that the annealed sample has a crystalline structure and lattice parameter like the bulk spinel ferrite (Fig. 2).

Electrical Properties of Co-Zn Ferrite Thin Film: Fig. 3 shows the sheet resistance of non-radiated and irradiated thin film of Co-Zn ferrite as a function of temperature. To improve the oxygenation and lower the resistivity of the sample we performed after deposition an additional annealing for $1 \mathrm{hr}$ at $600^{\circ} \mathrm{C}$. The film shows a cluster like island growth. The behavior of the sheet resistance with temperature can be divided into three regions. The first region is from room temperature to $95^{\circ} \mathrm{C}$ where the sheet resistance of the two films is the same. The second region ranges up to $170^{\circ} \mathrm{C}$ at which the resistance of irradiated sample is higher than the non-radiated one. Above $180^{\circ} \mathrm{C}$ the resistance of the non-irradiated in the third region has higher values. The two samples show a metallic behavior. The resistance increases sharply at $110^{\circ} \mathrm{C}$ for radiated sample and at $140^{\circ} \mathrm{C}$ for non-radiated sample.

The ferromagnetic-paramagnetic transition is correlated with the metal insulator transition with which produce a maximum in resistivity near Tc. So the resistivity maximum is expected in $180^{\circ}$ [which is near that for the bulk material $\left.\cong 173^{\circ} \mathrm{C}\right]^{[24]}$. A model can fit a plot of sheet resistance as a function of $\mathrm{T}$ that considering two parallel resistances one with high resistance corresponding to the film interface and the other with low resistance corresponding to the bulk of the film. The insulating behavior of the non-radiated film in the temperature range $\left(90-110^{\circ} \mathrm{C}\right)$ might be due to discontinuities that could arise from an island like growth mode and from the surface roughness of the film. 
The annealing process changes the electrical properties of the film which help in the growth of grains, lead to decrease the number of grain boundaries so decrease the resistivity and shift the peak resistance Tp. The intergranular transport can therefore be by tunneling or via metallic nano-contacts. In any case the intergranular resistance is much larger than the intrinsic resistance of the polycrystalline material itself which is about $6.5 \Omega$ at room temperature. The above discussion gives indication that the resistance will dominate by the grain boundary contribution. The hopping process of electrons between $\mathrm{Fe}^{2+}$ and $\mathrm{Fe}^{3+}$ is not direct process. The mobile electrons are captured in a grain boundary state, before tunneling to the adjacent grain. The film resistance shows metallic temperature dependence. The resistance of the bulk polycrystalline material, is about four orders of magnitude lower the film resistance $=28$ $\Omega$. The polycrystalline bulk material has a weakly semiconductor temperature dependence. The results indicate that a strong electron scattering occurs at the grain boundaries.

The non-radiated sample exhibit resistance maximum at $85^{\circ} \mathrm{C}$. The radiated sample does not show a peak resistance in this region. The $\mathrm{R}(\mathrm{T})$ curve has the expected metallic behavior without a lower temperature increase due to grain boundaries. The Curie temperature of the film can be changed by the internal pressure caused in the interface between the film and the substrate due to epitaxy stress. This internal stress affects $\mathrm{Fe}-\mathrm{O}$ bond length and $\mathrm{Fe}-\mathrm{O}-\mathrm{Fe}$ bond angle. The increase of the average radii of the A-site cations both cases the F-O bond to compress and the $\mathrm{Fe}-\mathrm{O}-\mathrm{Fe}$ angle to open in 180. This increase the covalent mixing between $4 \mathrm{dFe}$ orbital and those of $2 p$ from the oxygen, which enhancing the ferromagnetic double exchange interaction and thereby increasing $T_{c}$ and also the MI phase transition is affected by this internal stress.

According to the Mott's model when the charge carrier is not localized the conductivity is metallic, tending to a constant value at lower temperature i.e. The resistance of the given sample obey Mott's low ${ }^{[25]}$. The hopping electrons between $\mathrm{Fe}^{3+}$ and $\mathrm{Fe}^{2+}$ are scattered on the grain boundaries. When the temperature increases the grain boundaries become more mobile and scatter more hopping electrons so the resistivity increases. If the charge carriers are localized one expect a variable range hopping $\sigma=\sigma_{0} \exp \left(\frac{-B}{T^{\frac{1}{4}}}\right)$ at low temperature and excited to a mobility edge at high temperature $\sigma=\sigma_{0} \exp (\mathrm{E} / \mathrm{KT})$. When the material is actually metal, the formation of $\mathrm{Co}^{2+}, \mathrm{Co}^{3+}$ and $\mathrm{Fe}^{3+}$, $\mathrm{Fe}^{2+}$ ion at high temperature occurred with high and low spin states. These different charge states form a super lattice and then allow conductivity of metal type until they become distorted at higher temperatures.
As shown in Fig. 3 at high temperature the resistivity decreases after laser irradiation of the bulk material due to the following mechanism:

$$
\mathrm{Fe}^{3+}+\text { laser } \rightarrow \mathrm{Fe}^{2+}+\mathrm{e}
$$

This interaction will lead to an increase in the ratio $\mathrm{Fe}^{2+} / \mathrm{Fe}^{3+}$ lying at octahedral sites and in turn increase the hopping rate after irradiation, consequently, the electrical conductivity increases as shown in Fig. 4.

The annealing of the damages introduced by irradiation with pulsed laser indicates a substantial annealing of the resistivity at $200^{\circ} \mathrm{C}$ and $400^{\circ} \mathrm{C}$ and nearly complete annealing at $600^{\circ} \mathrm{C}$. The annealing make a distribution of charge defects.

Figure 5 shows the relation between the voltage across the sample and the sheet resistance, which give a straight line for both radiated and non-radiated sample. The current obtained from this is $0.01 \mathrm{~A}$, which fit well with that we established. This is the evidence that the measurements are right.

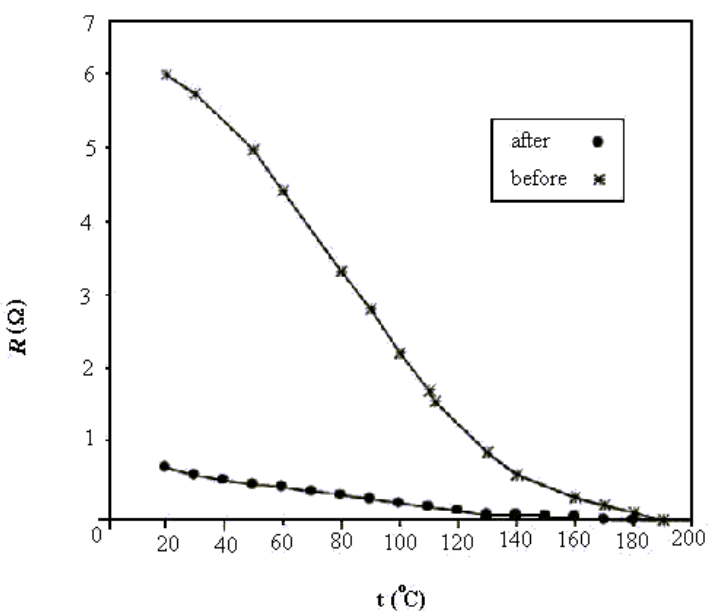

Fig. 4: Electrical conductivity of irradiated (dot) and non-radiated (star) samples vs temperature.

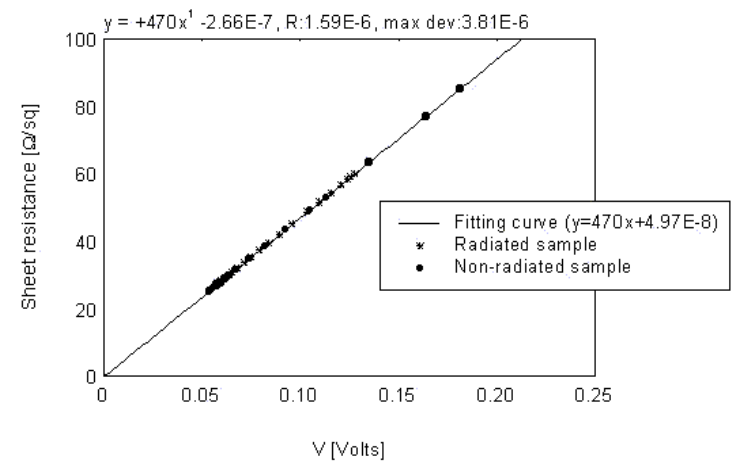

Fig. 5: Variation of sheet resistance as a function of voltage across the sample. 
In conclusion the two films radiated and nonradiated show a metallic behavior with temperature and the resistance is dominated by the grain boundary contributions. The maximum $\mathrm{R}$ is around the $\mathrm{T}_{\mathrm{c}}$ of the bulk material, which give the correlation between ferromagnetic to paramagnetic transition and Metal insulator phase transition. The annealing process decreases the resistance of the film due to the decrease of the number of grain boundaries. The hopping process of electrons between $\mathrm{Fe}^{2+}$ and $\mathrm{Fe}^{3+}$ which is dominant in the bulk material of ferrite, does not play a role in the thin film conduction because the hopping electrons are scattered on the grain boundaries. The resistance of the given sample obeys Mott's model which state that when the charge carrier are not localized the conductivity is metallic tending to a constant value at the low temperature region.

\section{ACKNOWLEDGMENT}

The author introduces his deep thanks to $\mathrm{Mr}$. Fawaz Aababnah from X-ray unit, Faculty of science, Yarmouk University, Jordan, for introducing facilities for measuring XRD for the given samples., and to $\mathrm{Mr}$. Samir Abdul-Rahman from Jordan University for his assistant in preparing the thin films.

\section{REFRENCES}

1. C. A. Carosella, D. B. Chrisey, Plubizz and E. Vittoria, J. Appl. Phys. 71 (10), 15 (1992).

2. S. B. Ogale and Rashmi Nawathey, J.Apple. Phys., 65 (1989).

3. D. S. Schmool, N. Keller, M. Guyot, R. Krishnan and M. Tessier. J. Apple. Phys. V86 No. 10 (1999).

4. D. S. Schmool, N. Keller, M. Guyot, R. Krishnan and M. Tessier. J. Magn. Magn. Mater, 195,291 (1999).

5. R. Krishnan, A. Lisfi, M. Guyot and V. Cagan. J. Magnetism and Magnetic Materials 147, L221 (1995).

6. Antony Ajan, Ramamuthy Acharya, Shivaprasad and S. N. Shingal, J. Apple. Phys. Vol. 83, No 11, 1 June (1998).
7. X. Sui, A. Singh, Y. Chen, D. N. Lambeth and M. H. Kryder, IEEE Trans. Magn. 31, 2776 (1995).

8. T. L. Hylton, M. N. Perker, M. Ullah, K. R. Coffeny. U. Unphress and J. K. Howard J. Apple. Phys. 75, 5960 (1994).

9. Li. R. Sinclair, S. S Rosenblum and H. Hayashi, J. Magnetism and Magnetic Materials 153, 246 1996).

10. 1.Y. Liu, A. Richard, S. S. Thomas, Z. S. Shan, S. H. Liou and J. Sellmyer J. Apple. Phys. 83,11 (1998).

11. M. F. Gillies, R. Coehoorn, J. B. A. Van Zon and D. Alders. J. Apple. Phys. Vol. 83. No 11, (1998).

12. C. M. Williams, D. B. Chrisey, P. Lubitz, K. S

13. Grabowiski and C. M. Cotell, J. Apple. Phys. 75, 1676 (1994).

14. Y. Suzuki et al. J. Appl. Phys. Lett. 68, 714 1996).

15. H. Mikami, Y. Nishikawa, and Y. Omato, Conf. Proc. Int. Conf. Ferrites7, 126 (1996).

16. P. J. Vander Zaag, J. M. Ruigrok and M. F. Gillies, Philips J. Res. 51 (1998).

17. W. A. Roshen, IEEE Trans. Magn. 26, 2880 (1990).

18. M. M. Amado, M. S. Rogalsk:, L. Guimaraes and B. Sousa, J. Appl. Phys. V. 83 No 11 (1998).

19. E. Katsnelson. J. Appl. Phys. 77 (9) (1995).

20. M. Nakano, K. Tomohara, J. M. Song and H. Fukunaga J. Appl. Phys. 87,9 (2000).

21. F. J. Cadieu, Li Chen, Blao Li and $\mathrm{T}$. Theodoropoulos. J. Appl. Phys. V. 87N11 (2000).

22. H. Q. Yin, J. S. Zhon and John B. Goodenough, J. Appl. Phys. 87, N9 (2000).

23. M. C. Smoak, P. A. Ryan and F. Tsni, J. Appl. Phys. 87,9 (2000).

24. E. Katsnelson J. Appl. Phys. 77, 9 (1995).

25. O. S. Josyulu and Sobhanadri, J. Phys. Stat. Sol., (4) 59 (1980). 